\title{
Reflexionando sobre la vejez a través del cine. Una aproximación incompleta
}

\author{
Sacramento Pinazo Hernandis \\ Departamento de Psicología Social \\ Universidad de Valencia
}

“El cine enseña a comprender, a pensar, a mirar. Entonces ¿cómo no va a ser un instrumento eficaz para la juventud?

En enseñar a mirar, está el sentido, también de aprender a pensar"

(Adolfo Bellido. Extraído de la entrevista que le hizo José L Barreda para la revista Cresol en 2001)

\section{resumen/alnstract:}

Existen en la sociedad muchos estereotipos y prejuicios en torno a las personas mayores, que se reflejan tanto en la vida cotidiana como en los medios de comunicación y también en el cine. En una sociedad audiovisual como la nuestra, la imagen es una importante fuente de conocimiento, y el cine se convierte en un excelente recurso didáctico para favorecer y motivar el proceso de enseñanza-aprendizaje; es una excelente herramienta con la que aprender acerca de la vida misma en general y las relaciones humanas en particular, donde observar y aprender acerca de las relaciones entre las personas en diferentes momentos de su ciclo vital; permite reflexionar, analizar, comprender, pensar, mirar y ofrece claves para ayudar a

las personas en su proceso de envejecer, mejorando su calidad de vida. En este artículo realizamos una selección de películas que lleven a profundizar en el conocimiento de las personas mayores y las cuestiones relevantes para ellos en sus dinámicas vitales y en su relación con otras generaciones; películas que muestran el mejor modo de envejecer, el denominado envejecer en positivo, para ayudar a reducir los estereotipos negativos acerca de la vejez, seleccionando algunas de ellas; películas que muestren el envejecimiento activo y solidaridad intergeneracional.

A lot of stereotypes and prejudices have been created around old people in our modern society; they're reflected in every day life as well as in mass media and also in films. In our audio visual society image is a very important source of knowledge, and films are anoptimal educational resource for improvement and motivation in teaching and learning processes. Films are a wonderful tool for learning about life in general and specially about relationships across lifespan. Films allow to reflect, to analyse, toknow, to think, and to examine a lot of situations and offers keys that may help people in their growing processes, improving their quality of life. In this article, we have made a selection of films to deepen people's knowledge about older people, heir main questions in their life and relationships between generations; we have made a selection of films that show the best way to grow up-so-called successful ageing, active ageing and solidarity tween generations-, that may help reduce negative stereotypes around the elderly.

\section{palabras clave/keywords:}

Cine, personas mayores, envejecimiento activo, cambio de estereotipos, solidaridad intergeneracional.

Films, elderly, active ageing, sterotypes reduction, intergenerational solidarity. 


\section{Introducción}

El año 2012 ha sido declarado por el Parlamento Europeo, Año del Envejecimiento Activo y la Solidaridad Intergeneracional con el objetivo de concienciar a la población europea a envejecer mejor desarrollando programas y proyectos en torno al envejecimiento activo, saludable, productivo y con éxito. Porque las poblaciones están envejeciendo. Los datos actuales dicen que en la Unión Europea (UE-27), cerca de 75 millones de habitantes tiene más de 65 años. En el caso de España, vivimos en un país envejecido en el que la población de los de más edad tiene un peso relativo y absoluto mayor. En España, las personas mayores representan más del $17 \%$ de la población, que en números concretos son 8.092.853 personas -datos del Instituto Nacional de Estadística (INE, 2012) basados en el Padrón de 2011-, siendo el envejecimiento desigual en función del género: aproximadamente 4 millones y pico de mujeres y 3 millones y pico de hombres. Las proyecciones del INE para el 2050 señalan que España alcanzará los 53 millones de personas y la población de 64 ó más años pasará del $17 \%$ actual al $31.9 \%$ del total de la población. Este 'envejecimiento del envejecimiento' es consecuencia de varias circunstancias: un aumento de la esperanza de vida al nacimiento -76.9 años para los hombres y 83.4 años para las mujeres que se espera que aumente hasta 84.3 años en los varones y 89.9 años en las mujeres en 2048-.

A nuestro entender, el envejecimiento poblacional obliga a evaluar las nuevas necesidades sociales y repensar las políticas y los programas que deberán favorecer el envejecimiento activo y saludable y prevenir o retrasar la dependencia, y además ser creativos y diseñar programas que permitan la participación social de los ciudadanos más mayores y de todas las edades $y$ en donde ellos sean los protagonistas y no meros espectadores. Y siempre teniendo presente que no existe un único modo de envejecer sino que existen grandes diferencias intra e interindividuales por su multidimensionalidad en un proceso donde ocurren pérdidas y también ganancias. Los cambios que pueden darse durante la vejez dependerán mucho de las características individuales de cada sujeto, del microsistema, grupo/sociedad al que pertenece, del nivel meso o macrosistema, pero sobre todo del modo de afrontar dichos cambios.

Mantenerse activo mientras se envejece es la clave para afrontar los cambios del envejecimiento. Entender el envejecimiento activo como "el proceso que implica envejecer con buena salud, óptimo funcionamiento físico, cognitivo, emocional y motivacional, además de con una alta participación social" (OMS, 2002), significa que un buen envejecer se logra a partir de una buena salud, un buen funcionamiento intelectual, afecto y compromiso con los demás. Envejecer activamente significa vivir bien cada ciclo de la vida: mantener hábitos saludables (previniendo la enfermedad), optimizar el funcionamiento físico y cognitivo, regular las emociones, controlar la vida y participar activamente con la familia y en la sociedad. De esta forma se consiguen múltiples beneficios como la mejora de la autoestima, el mantenimiento de hábitos de vida saludables, se evita el aislamiento y se retrasa la dependencia. Algunos autores (Triadó y Villar, 2008) lo han llamado envejecer en positivo, $y$ supone la búsqueda de la realización personal, continuar con un crecimiento personal viviendo experiencias positivas de satisfacción vital. 
Vinculado a este concepto de envejecer de un modo satisfactorio se encuentra el Modelo de Optimización Selectiva con Compensación (Baltes y Baltes, 1990) que recoge la idea básica de desarrollo como un proceso de adaptación selectiva. Por un lado, en el proceso de maduración, las personas vamos seleccionando, escogiendo determinadas trayectorias, situaciones y personas en nuestro entorno y dejando otras; una vez elegidas ciertas trayectorias, optimizamos los recursos a nuestro alcance; y compensamos adquiriendo nuevos medios que sustituyen a los que se han perdido o bien sustituimos las metas previas por otras alcanzables más fácilmente.

También vinculados a la etapa de la madurez y vejez encontramos en la literatura científica tres conceptos de la psicología del ciclo vital de gran interés para el tema que nos ocupa: son los de integridad, sabiduría y generatividad. Erik Erikson habló del crecimiento y la realización personal en la última etapa del ciclo vital: "El fruto de los siete estadios sólo madura gradualmente en la persona que está envejeciendo, que se ha ocupado de las cosas y de la gente, y se ha adaptado a los triunfos $y$ a los desengaños de ser, por necesidad, el que ha dado origen a otros y ha producido objetos e ideas. Para expresar este resultado no conozco mejor palabra que 'integridad'” (Erikson, 1971: 113). Muchas personas se vuelven más reflexivas conforme envejecen; la etapa final de la vida se torna un momento de revisión de la vida pasada, de reinterpretación y reintegración de los acontecimientos vividos y de la manera que se ha tenido de superarlos. A partir de dicha revisión, el individuo necesita aceptar que ha merecido la pena lo vivido, que su vida ha tenido sentido. Si la persona logra el sentido de integridad, desarrollará un estado de serenidad que le permitirá afrontar sin temor los últimos años de su vida y alcanzar la sabiduría (Baltes y Smith, 2003; Baltes y Satudinger, 2000), una suerte de conocimiento experto de los aspectos prácticos de la vida, fruto de la acumulación de conocimiento, experiencia y reflexión. La sabiduría conlleva llevar el buen juicio al conducir la propia vida, desarrollar la comprensión por medio de la experiencia y la capacidad para aplicarla a los asuntos importantes (Baltes, Smith, Staudinger y Sowarka, 1990). Además, la integridad del Yo está muy relacionada con el envejecimiento activo (autores como Hannah, Domino, Figueredo y Hendrickson, 1996, por ejemplo, han aportado evidencias empíricas de esta relación).

El otro concepto, la generatividad o "preocupación por establecer y guiar a la nueva generación” (Erikson y Erikson, 1987)- traducida en actos de cuidado-es lo que define a las personas en su etapa de madurez. La generatividad abarca un amplio abanico de actividades en el contexto familiar, social y cultural. En el contexto familiar, por ejemplo, el cuidado a familiares dependientes, la ayuda a los hijos y el cuidado a los nietos. En el contexto social y cultural, se hace referencia al cuidado a otros a través del voluntariado, la participación cívica, social o política, el asociacionismo, la participación en programas educativos, formativos y culturales y la participación en programas intergeneracionales. La generatividad se relaciona con toda actividad en la que el individuo se halla involucrado en la promoción directa del desarrollo de otros. La interdependencia entre la persona cuidada y el cuidador, entre la generación más joven y la de más 
edad, caracteriza las relaciones adultas generativas: el adulto transmite los valores a la nueva generación, actúa como mentor y modelo. Las personas mayores tienen una experiencia de vida vivida valiosa de la que pueden aprender otras generaciones y de la que se pueden beneficiar todos.

Los Congresos Estatales de Personas Mayores (especialmente el de CEOMA, celebrado en 2009), -inspirados en las recomendaciones realizadas por la II Asamblea Mundial del Envejecimiento, los Planes Gerontológicos Nacional y Autonómicos-, también destacan el envejecimiento activo y las relaciones intergeneracionales como importantes áreas de atención y desarrollo. Como ejemplo de ello, recordamos una de las recomendaciones de la II Asamblea Mundial del Envejecimiento:

(Cuestión 5: Solidaridad Intergeneracional) "La solidaridad a todos los niveles es fundamental para el logro de una sociedad para todas las edades. A nivel de familia y comunidad, los vínculos intergeneracionales pueden ser beneficiosos para todos. Fortalecer la solidaridad mediante la equidad y la reciprocidad entre las generaciones; promover, mediante la educación, la comprensión del envejecimiento como una cuestión que interesa a toda la Sociedad".

En el Informe al Parlamento Europeo sobre el futuro demográfico de Europa 2007/2156 (INI), publicado en 2008, se hacen interesantes consideraciones a este respecto:

(C) Considerando que la media europea de la tasa de dependencia por vejez (número de personas de más de 65 años dividido por el número de personas de edades comprendidas entre los 14 y los 65 años) pasará del 25\% en 2004 al $53 \%$ en 2050.

(68) Recuerda la enorme contribución que aportan las personas mayores a la cohesión social y económica, y que $s u$ participación activa en las relaciones de solidaridad familiar y entre generaciones fortalece la redistribución de los recursos existentes dentro de la cadena familiar; considera por otra parte, que se debe facilitar y fomentar su participación en actividades de voluntariado dentro de la economía social recurriendo para ello a contrapartidas financieras; opina, por último, que su consumo de bienes y servicios y de prestaciones de ocio, cuidados y bienestar constituye un sector económico en fase de expansión y una nueva fuente de riqueza denominada 'oro gris'; pide, así pues, a los Estados miembros que fomenten y desarrollen la participación económica y social de las personas mayores, velando particularmente por su bienestar físico y para que disfruten de unas buenas condiciones de vida social y financiera.

(69) Pide a los Estados miembros que promuevan la consulta de las personas mayores a fin de mantener la solidaridad entre las generaciones y que faciliten su participación en actividades voluntarias de carácter educativo, cultural o empresarial.

(79) Insta a los Estados miembros a que promuevan los proyectos intergeneracionales en los que las personas mayores trabajan junto con los jóvenes para compartir capacidades y adquirir nuevos conocimientos; pide a la Comisión 
que facilite el intercambio de buenas prácticas en este ámbito.

Conscientes de la importancia de los medios de comunicación social como creadores de opinión, y a iniciativa del Consejo de Personas Mayores de Vizcaya, hace un par de años la Diputación Foral de Vizcaya reunió a técnicos de la Diputación con periodistas y representantes de diferentes asociaciones de personas mayores -entre las que se encontraban Nagusilán, Nagusiak y Hartu Emanak-, con el fin de redactar un informe acerca de Las personas mayores y los medios de comunicación (2011). Iniciaron un grupo de trabajo para hacer un diagnóstico sobre el papel de las personas mayores en los medios de comunicación y redactar algunas recomendaciones y propuestas.

El texto antecitado comienza su introducción diciendo (2011: 5): “...no podemos permitir que la vejez siga siendo vista de forma negativa, ni que se proyecte una imagen irreal de la misma”.

En su página 11, hablando de la promoción de una imagen de la vejez y el envejecimiento más positiva, concluyen con un diagnóstico y unas recomendaciones posteriores:

(Diagnóstico) Ocio y consumo parecen lo único que se valora de las personas mayores. Esta imagen estereotipada, y por tanto negativa, está haciendo que se pierda socialmente la aportación de toda una generación de personas mayores. El envejecimiento en el siglo XXI y el papel de las personas mayores será diferente al del siglo pasado, pero, entre tanto, el vértigo de los cambios sociales y tecnológicos actuales hace que la correa de transmisión entre ge- neraciones y la aportación de la experiencia humana y vital no se aproveche.

(Recomendaciones) Sería interesante primar la imagen activa de las personas mayores frente a imágenes no tan positivas. Promover desde los medios programas o espacios con una dimensión intergeneracional, en los que participen miembros de distintas generaciones, sobre temas de interés común, que ayuden a "acortar distancias" entre generaciones, a entenderse mutuamente, a construir juntos una sociedad para todas las edades, una sociedad más justa y cohesionada. Hacer un reconocimiento público del valor de la contribución de las personas mayores en la construcción de la actual sociedad (desarrollo industrial, recuperación de la democracia, etc.). Elegir modelos de envejecimiento activo, personajes que han sido relevantes durante toda su vida. Esta gente podría ser imagen de las campañas de promoción publicitarias. Destacar el valor del papel de las personas mayores como agentes sociales en el ámbito del voluntariado.

Y finalmente, como Decálogo, que aparece en la página 18, dice, entre otros puntos:

7. los medios de comunicación social deben participar en la tarea de reivindicar un envejecimiento activo y dar a las personas mayores el papel que les corresponde en la sociedad, haciendo ver que el envejecimiento activo es cosa de todos, desde los menores hasta las personas mayores. Los medios de comunicación tratarán de ayudar a crear una imagen de las personas mayores más 
acorde a la realidad del momento: personas activas, poseedoras de un caudal de experiencia e información relevante para la sociedad.

8. las personas mayores, de forma individual o colectiva, necesitan desarrollar un proceso de formación y sensibilización para llegar a "empoderarse" y actuar como verdaderos actores sociales, capaces de colaborar en la construcción de una sociedad para todas las edades.

Por último, no quiero olvidarme de mencionar el capítulo de Loles Díaz Aledo en la publicación de la Sociedad Española de Geriatría y Gerontología, Guía Práctica del Buen Trato a las personas mayores. Entre otras muchas cosas interesantes, destacamos estos párrafos-resumen con los que comienza su texto (Díaz, 2011: 109):

"Mostrar el envejecimiento como una etapa más de la vida rescatando los valores que encarnan las personas mayores; incluir a las personas mayores en todo tipo de informaciones, en plano de igualdad con los demás; evitar el lenguaje peyorativo, paternalista y discriminatorio que contribuye a reforzar los estereotipos sociales; propiciar el tratamiento de los temas con una perspectiva intergeneracional y darles voz como ciudadanos/as independientes y protagonistas de sus vidas."

"Las personas mayores del siglo XXI piden a los periodistas que salgan a la calle y miren queriendo ver. Que se acerquen a su realidad porque están en todas partes y junto a las demás generaciones."

"No caer en el paternalismo y huir del dramatismo y el sensacionalismo y en todo caso contextualizar las informaciones mostrando las causas y circunstancias de una situación."

Todas estas medidas pretenden que la sociedad cambie la visión que tiene sobre la vejez y sobre las personas mayores, dado que existen muchos estereotipos y prejuicios en torno a las personas mayores, que se reflejan tanto en la vida cotidiana y en los cuidados, como en los medios de comunicación... y el cine.

Los estereotipos son falsas concepciones en el acercamiento a un grupo social. Son ideas sobre las características personales de la mayor parte de las personas que forman parte de un grupo determinado; son inexactos y generalmente negativos y favorecen la aparición de conductas discriminatorias. En el caso del estereotipo negativo de la vejez, suele dar lugar a comportamientos edadistas, un tipo más de discriminación grupal, como lo son el racismo o el sexismo. En 1969 Butler definió el término edadismo (ageism) refiriéndose a los estereotipos sistemáticos y discriminatorios contra las personas de edad por el simple hecho de ser mayores y que se reflejan en conductas como el desdén, el desagrado, el insulto o, simplemente, evitando la cercanía y el contacto físico. Los estereotipos negativos acerca de la vejez en las sociedades occidentales afectan de manera especial a las mujeres.

Las interpretaciones negativas y peyorativas de la vejez son las más comunes y asocian vejez con deterioro, inutilidad, aislamiento, improductividad y desvinculación de la persona mayor respecto a los intereses sociales y relacionales comunitarios - "Se jubila a los que ya no sirven" o "La jubilación es la antesala de la muerte”, entre otras, son algunas de estas falsas ideas- .Si 
bien es cierto que la salud subjetiva y la objetiva se van deteriorando con la edad, este deterioro no es brusco y sólo por cumplir 65 años no se produce ningún cambio ni se entra en situación de incapacidad o dependencia.

Los estereotipos negativos que se atribuyen a las personas mayores minan su autoconcepto, la imagen que la persona mayor tiene de sí misma e incluso las expectativas que tiene con respecto a la vejez. Una imagen negativa de la vejezprovoca rechazo $-\mathrm{Na}$ die se quiere morir joven pero nadie quiere que le llamen "viejo"- pero no sólo rechazo a la persona mayor sino también rechazo al mismo proceso de envejecer.

Los medios de comunicación a lo largo de su historia también han proyectado estos mismos estereotipos de la vejez (homogeneidad, pasividad, discapacidad, dependencia, fragilidad, improductividad, senilidad, antigüedad, 'fuera de onda'...). Para la publicidad, el grupo que actúa como referente de la tradición y experiencia son las personas mayores. Paseando por la historia reciente de la publicidad española podemos encontrar abundantes ejemplos donde éstos y el escenario rural han sido imagen y escenario de célebres campañas. En ella se muestra una imagen de las personas mayores acompañadas de 'marcadores de edad' que definen a la 'auténtica vejez' como el bastón, el pelo blanco la calvicie, el moño, la dentadura postiza, el refajo y el delantal...pese a que esta imagen no corresponde a la de las personas mayores de 65 años que uno se encuentra habitualmente por las calles (valga como ejemplo, la Abuela de la Fabada...).

Sin embargo, al margen de campañas como la nombrada anteriormente, la presencia de personas mayores en la publicidad espa- ñola no ha sido hasta ahora muy habitual (Ramos y Carretón-Ballester, 2012). La comunicación publicitaria habitualmente se piensa, se dirige y diseña para un público diana muy joven. Pero en este artículo nos vamos a centrar sólo en el cine donde, a menudo, tampoco se refleja la realidad, tal y como dice Aurea Ortiz (2006: 239):

"No hay sitio para la gente mayor, o, si lo hay, es en una esquina del plano, sin molestar mucho, y sólo si su presencia contribuye a ese mensaje general e implacable que indica que 'el mundo es de los jóvenes'. Rara vez un personaje mayor será el protagonista de un film; en todo caso, será un secundario, de mayor o menor importancia, al servicio del personaje principal. Por ejemplo, en Ginger y Fred (Ginger e Fred, dir: F. Fellini, 1986), a través de la anciana pareja de bailarines protagonistas, se revela la crueldad de esta grotesca sociedad del espectáculo y la frivolidad, que no respeta nada ni a nadie. Ni a la edad. De vez en cuando, gente mayor, ancianos y jubilados son el soporte de alguna película que suele plantearse la reflexión en torno al paso del tiempo y qué sucede cuando se envejece. Paseando a Miss Daisy (Driving Miss Daisy, dir: B. Beresford, 1989) y Tess y su guardaespaldas (GuardingTess, dir: H. Wilson, 1994) hablan de estas cuestiones fijando el conflicto entre dos personajes antagónicos que se ven obligados a convivir. En Mrs. Henderson presenta (Mrs. Henderson presents, dir: S. Frears, 2005), JudyDench es una emprendedora y atípica mujer viuda que a sus 69 años se inicia en los negocios, en este caso, comprando un teatro en el Soho. Cleopatra (Cleo- 
patra, dir: E. Mignogna, 2003) sitúa como eje de la acción a una jubilada que decide cambiar de vida a pesar de la edad y de la presión social."

Diferentes películas muestran esos estereotipos negativos a los que antes hemos aludido: Umberto D. (Umberto D.,dir: V. de Sicca, 1952) -el protagonista, un funcionario jubilado con una minúscula pensión, con una vejez desdichada e insignificante, vive en soledad y con la única compañía de su perro-; El Gatopardo (Il Gattopardo, dir: L.Visconti, 1963) -profunda reflexión acerca de la decadencia-; A propósito de Schmidt (About Schmidt, dir: A. Payne, 2002) -el protagonista, recién jubilado, sin saber qué hacer con su tiempo y con su vida-; Muerte en Venecia (Morte a Venezia, dir: L.Visconti, 1971) -homenaje a la belleza perfecta, pura y plena, inalcanzable, propia solo de la juventud, junto a la decadencia y el paso del tiempo; y La Balada de Narayama (Narayama Bushi-Ko, dir: S. Imamura, 1983) -que narra el drama de una mujer de 70 años al ver cerca el momento, no de la muerte, sino de su abandono forzoso en la montaña-. Pese a ser buenas películas la mayor parte de ellas, son buenos ejemplos de estos estereotipos negativos a los que hacíamos referencia.

Otras películas han intentado reflejar el ansia del ser humano por alejar la vejez lo más posible, la búsqueda de la eterna juventud, el pacto con el tiempo del que hablaba Oscar Wilde (El retrato de Dorian Gray, 1890) - "La tragedia de la vejez no es que uno sea viejo, sino que una vez fue joven" cita en el texto-. Películas que muestran esa búsqueda del Jardín de las Hespérides, Shangri-La, o el manantial de la juventud son Horizontes perdidos (Losthorizon, dir: F. Capra, 1937),Cocoon (Cocoon, dir: R.
Howard, 1985) y Cocoon: el retorno (Cocoon: the return, dir: D. Petrie, 1988).

\section{Justificación del tema de estudio}

En una sociedad audiovisual como la nuestra, la imagen es una de las principales fuentes de conocimiento, y el cine se convierte en un excelente recurso didáctico para favorecer y motivar el proceso de enseñanza-aprendizaje. El cine presenta ventajas frente a otras propuestas formativas, por ejemplo, que el aprendizaje puede ser más duradero por el uso de las imágenes o que puede ser muy atractivo para todos los públicos, aunque también presenta algunos inconvenientes como el hecho de que no muestra la realidad sino una representación de la misma, desde un enfoque o visión particular, la del director/a de la película quien eligió los códigos visuales, el uso de luz y color y los códigos sonoros, el montaje, cómo rodar las tomas, escenas y secuencias, cómo situar la cámara (neutra, picada), cómo moverla (travelling, panorámica) y qué profundidad de campo dar... Algunas películas sirven para ahondar en temas psicológicos o psicopatológicos ayudando al espectador a entender mejor diferentes comportamientos humanos acercándole a las emociones y sentimientos de los protagonistas. Memento, es un ejemplo de ello (dir: C. Nolan, 2000), en ella, el protagonista padece amnesia anterógrada y por ello no puede recordar nada posterior a su accidente. El espectador experimenta la misma sensación debido al montaje a la inversa que el director hace de los episodios. O Con la muerte en los talones (North by Northwest, dir: A. Hitchcock, 1959), en donde el objetivo del director parece ser que el espectador se identifique totalmente con el protagonista a través de las situaciones absurdas, exageradas e incontrolables 
que le hace vivir. O los flashbacks que utiliza Richard Eyre en su película Iris (2001), combinando presente y pasado, para mostrarnos cómo la protagonista va perdiendo los rasgos que han definido su vida tan ligada al mundo de las letras para ser cambiados por la afasia, ecolalia, palilalia, anomia, disgrafía, jergafasia. Otras películas ahondan en la interpretación psicológica de las acciones de los protagonistas: qué hacen, qué sucede a lo largo de la narración, qué relaciones tienen, los conflictos o interacciones que se dan entre ellos, los momentos vitales en los que se encuentran, la historia de sus vidas. Aquí no podemos olvidarnos de citar al maestro del drama psicológico, Ingmar Bergman (1918-2007) y su particular forma de describir al ser humano, sus pasiones, sus dramas, sus relaciones. Entre otras -relacionadas con el tema que nos ocupa- merece la pena destacarse: Fresas salvajes (Smultronstrället,1957) y la revisión de vida al final de la misma y con la excusa de un viaje; El séptimo sello (Detsjundeinseglet,1957) sobre la llegada de la muerte; Sonata de otoño (Höstsonatten, 1978), Secretos de un matrimonio (Scenerurettäktenskap, 1974) y -su continuación- Saraband (2003), sobre las relaciones familiares y de pareja o las escenas de vida conyugal en el amor maduro.

El cine es, pues, una excelente herramienta con la que aprender acerca de la vida misma en general, y las relaciones humanas en particular; permite reflexionar, analizar, comprender, pensar, mirar y ofrece claves que ayudan a las personas en su proceso de envejecer, mejorando su calidad de vida. El cine permite ver en la pantalla reflejada la propia vida, a la vez que también permite observar ejemplos de comportamientos $\mathrm{y}$ respuestas a situaciones vividas o no, empatizar con los protagonistas, acercarse a su mundo, compararlo con el propio, el cercano, el vivido, reactivar recuerdos o "experienciar" en cabeza ajena. El cine modifica nuestra configuración de la experiencia diaria porque aumenta nuestra capacidad de tener experiencias en el ámbito de la virtualidad real, lo cual permite disfrutar del mundo desde una nueva luz y ver así las cosas de una forma totalmente distinta (Castells, 2000). En el cine podemos observar y aprender acerca de las relaciones entre las personas en diferentes momentos de su ciclo vital. Siguiendo nuevamente a Ortiz, 2006: 241):

"Uno de los ejemplos más hermosos de solidaridad intergeneracional lo ofrece Dersu Uzala (dir. A. Kurosawa, 1975), narración de la amistad entre dos hombres muy distintos que se encuentran en condiciones extremas, y en la que el mayor enseñará al más joven donde se encuentra lo esencial y el verdadero sentido de la existencia. Otros ejemplos son el del proyeccionista ciego que le enseña la luz de la vida y la dignidad a su pupilo en Cinema Paradiso (dir: G.Tornatore, 1989); Fernando Fernán Gómez encarnando el saber, la libertad y la curiosidad en La lengua de las mariposas (dir: J.L. Cuerda, 1999); el paciente maestro que le muestra a su pupilo el difícil camino de la vida en Primavera, verano, otoño, invierno... y primavera (Bom yeoreum gaeul gyeoulgeurigo bom, dir: Kim Ki-Duk, 2003); el ejemplo de libertad y compromiso que ofrece el personaje más mayor a su amiga en la edad madura, en Tomates verdes fritos (Fried Green Tomatoes, dir: J.Avnet, 1991); la impagable madre interpretada por China 
Zorrilla en Conversaciones con mamá (dir: S. C. Oves, 2004); la lección de amor que, de sus padres ancianos, hereda el protagonista de El hijo de la novia (dir: J.J. Campanella, 2001); el anciano africano que viaja a Estados Unidos para buscar a sus familiares y recordarles su identidad en Little Senegal (R. Bouchareb, 2001); las enseñanzas vitales que aprende el muchacho protagonista de El secreto de los McCann (Secondhand Lions, dir: T. MacCanlies, 2003). Son películas muy diversas, tanto por el tono como por la historia o la estética, pero todas ellas centran el sentido de la narración en las enseñanzas que una generación mayor puede ofrecer a las otras."

A veces, hay una solidaridad intergeneracional manifestada por un aprendizaje y una enseñanza, en un quid pro quo, aprendizaje en dos direcciones: de la persona mayor al joven y del joven a la persona mayor. En Million Dollar Baby (dir: C. Eastwood, 2004), el protagonista y maestro de la aprendiza de boxeadora, es quien le enseña y le forma, quien le transmite valores, quien hace de mentor, y es también quien la cuida hasta su final. En Para que no me olvides (dir: P. Ferreira, 2005) será el nieto quien se encargará de recoger y completar la memoria de su abuelo, cada vez más sumido en la enfermedad de Alzheimer. Siguiendo a Ortiz (2006: 241): "La presencia de personas mayores en las ficciones se relaciona muchas veces con la posibilidad de elaborar personajes que son depositarios de cierta sabiduría que su experiencia les ha dado, y están en disposición de dejar un legado moral o sentimental que los protagonistas han de recoger como una lección de vida. Este planteamiento se inserta ha- bitualmente en un ámbito familiar, pero no siempre; puede ser otra persona mayor la que actúa como portadora del conocimiento".

Ed Wood (dir. T. Burton, 1994) se centra en el emotivo vínculo que establecen dos desafortunados artistas, un joven y desastroso director y un viejo Bela Lugosi en decadencia. En Seven (dir: D. Fincher, 1995) dos policías, un mentor pesimista y su optimista alumno viajan a los infiernos de este mundo en un viaje sin retorno posible. El sentido de la vida también puede buscarse en la relación con los hijos adultos y en la gestión de los cuidados o los afectos o en comprobar el grado de satisfacción con sus vidas, como en El estanque dorado (On Golden pond,dir: M. Rydell, 1981), Todos están bien (Stanno tutti bene, dir: G.Tornatore, 1990), y su remake Todos están bien (Everybody's fine, dir: K. Jones, 2009) y Dejad paso al mañana (Make way for tomorrow, dir: L. McCarey, 1937) (adaptado de Genovard y Casusellas, 2005: 17 y 18). Otras películas que hablan de la relación intergeneracional y no queremos olvidar mencionar son: Capitan Abu Raed (Captain Abu Raed, dir: A. Matalga, 2007), Charlie y la fábrica de chocolate (Charlie and the chocolate Factory, dir: T. Burton, 2005), Cuentos de Tokio (Tokio monogatari, dir: Y. Ozu, 1953), Dame diez razones (Ten items or less,dir: D. Silberling, 2006), El abuelo (dir: JL. Garci, 1998), El bosque de luto (Mogari no mori, dir: N. Kawase, 2007), El color del paraíso (The color of Paradise, dir: M. Majidi, 1999), El mundo de Marty (Le monde de Marty, dir: D. Bardiau, 2000), El verano de Kikujiro (Kikujiro no natsu,dir: T. Kitano, 1999), El viento (dir: E. Mignona, 2005), Esencia de mujer (Scent of a woman, dir: M. Brest, 1992), 
Gran Torino (dir: C. Eastwood, 2008), Kolya (dir: J. Severák, 1996), Kotch (dir: J. Lemmon, 1971), La casa de mi abuela (dir: A. Aliaga, 2006), Mil años de oración (A thousand years of goodprayers, dir: W. Wang, 2007), Pequeño cherokee (The education of Little tree, dir: R. Friedenberg, 1997), Sang Woo y su abuela (Theway home, dir: L. Jeong-Hyangh, 2002), Un poco de chocolate (dir: A. Aramaio, 2008), Un toque de canela (Politiki koucina, dir: T. Boulmetis, 2003), Y tú quién eres? (dir: A. Mercero, 2007).

Un eje de análisis en las películas sobre la vejez -según Genovard y Casulleras, 2005se refiere a la dimensión de poder y su utilización ética que detentan los mayores. Así, encontramos personas mayores que utilizan su poder para hacer el bien como el Gandalf de El señor de los anillos (dir: P. Jackson, 2001, 2002, 2003) o Saruman quien en la misma película usa el poder para hacer el mal, como otras personas mayores que aparecen en la trilogía de El Padrino (dir: F.F. Coppola, 1972), Ciudadano Kane (dir: O. Welles, 1941), La huella (dir: J.L. Mankiewicz, 1972), Reservoir Dogs (dir: Q. Tarantino, 1992), La tapadera (dir: S.Pollack, 1993), Hannibal (dir: R. Scott, 2001) o Camino a la perdición (dir: S. Mendes, 2002). En cambio, la persona mayor sin poder es un ser insignificante que no puede ejercer ningún daño o, en todo caso, sólo puede ejercer el bien, como el protagonista de Una historia verdadera (dir: D. Lynch, 1999). La jubilación también suele suponer un importante cambio para la persona (habitualmente, representado en un hombre) que ha hecho del trabajo su identidad. La jubilación suele ser mostrada como un acontecimiento negativo en la vida de las personas que supone una crisis vital; esto lo vemos en películas como A propósito de Schmidt (dir: A. Payne, 2002). En cambio, el protagonista de Lugares comunes (dir: A. Aristaraín, 2002) es capaz de encontrar un sentido a su existencia retomando de forma nostálgica los sueños revolucionarios de su juventud.

El uso del cine como recurso educativo ha sido recomendado desde hace tiempo por la UNESCO (1960: 19): “Asimismo conviene utilizar los grupos ya existentes (familias, organizaciones de juventud, etc.) haciéndoles participar en la crítica de la prensa, de los programas de radio, de televisión. Los educadores deben utilizar los grandes medios de información de la misma manera que utilizan los libros, puesto que esos medios se dirigen al público en general. ....... Los medios audiovisuales (carteles, películas fijas, exposiciones modernas, radio y televisión) son instrumentos de educación muy útiles.”...... “Estos medios permiten colmar el foso que media entre los conocimientos del especialista y el hombre de la calle, favorecen los cambios de impresiones sobre las ideas, la vida y la mentalidad entre diferentes grupos de ciudadanos y entre países, y enriquecen el conocimiento que tenemos de nuestro medio, del mundo en general", respaldada actualmente por la Ley Orgánica de Educación (Ley Orgánica 2/2006, de 3 de mayo) -arts. 17, i- "Iniciarse en la utilización, para el aprendizaje, de las tecnologías de la información y la comunicación desarrollando un espíritu crítico ante los mensajes que reciben y elaboran”, y por la Ley del Cine (Ley 55/2007, de 28 de diciembre) -disposición adicional séptima-Acceso de los productos cinematográficos y audiovisuales al sistema educativo: "Las Administraciones Públicas, en el ámbito de sus respectivas com- 
petencias, promoverán la accesibilidad de los productos cinematográficos y audiovisuales al sistema educativo a través de programas de formación, de manera que sus contenidos puedan también quedar integrados en aquél”, y actualmente se utiliza mucho en las Universidades para la formación en competencias exigida en los nuevos grados derivados del Espacio Europeo de Educación Superior (Estrategia de Lisboa, más conocida como Plan Bolonia, firmado en 1999): Se trata de introducir al alumno en una experiencia educativa que le permita desarrollarse en todas las dimensiones de la persona, Saber (conocimientos), Saber hacer (habilidades y competencias), Saber ser (actitudes).

Por todo ello, para el estudio que aquí presentamos, nos planteamos realizar una selección de películas que nos permitiesen alcanzar varios objetivos:

a) utilizando las imágenes que el cine nos muestra, profundizar en el conocimiento de las personas mayores y las cuestiones relevantes para ellos en sus dinámicas vitales y en su relación con otras generaciones;

b) destacar aquellas películas que muestran el mejor modo de envejecer, el denominado envejecer en positivo, para ayudar a reducir los estereotipos negativos acerca de la vejez, seleccionando algunas de ellas;

c) ahondar en el concepto "envejecimiento activo y solidaridad intergeneracional" tomando como ejemplo algunas películas que lo muestran;

d) además, y por último, la selección de películas y su clasificación pueden servir para el uso de las películas y documentales como elemento educativo, tanto de los profesionales sociosanitarios en general como de los psicogerontólogos en particular, pero también de la sociedad en su conjunto (las propias personas mayores, y todas las que están en proceso de madurez desde los niños y jóvenes hasta los de mediana edad).

Plantear este artículo no ha sido nada fácil pues quizás habría requerido hacer una búsqueda exhaustiva de todas las películas producidas que tuviesen como protagonistas a personas mayores para poder, después, seleccionar aquellas que cumplieran un criterio preestablecido y que mejor pudieran representar los temas que queríamos desarrollar en el artículo. Finalmente, nos decantamos por hacer una buena, aunque incompleta, larga -pero no conclusa- búsqueda, para pasar posteriormente a realizar en ella una selección y una clasificación temática. Esperamos que el lector aprecie la lista y las recomendaciones anejas a ella, y también esperamos que esto sea el comienzo de una lista abierta y creciente, y que los diferentes lectores de este artículo puedan enviar sugerencias de películas y recomendaciones de visionado.

\section{Procedimiento}

El cine es un reflejo de la realidad que lo origina pero también puede llegar a ser un creador de imágenes culturales y representaciones. A través de este artículo se pretende explorar parte de la imagen de la vejez en el cine, a partir de una serie de films seleccionados que presentan una imagen positiva del envejecimiento y que no caen en lo peyorativo o burlesco, y tampoco en el sentido patológico de la vejez como pueden ser temas relativos a las demencias y otras enfermedades. 
No se trata de ofrecer un análisis completo de la imagen que el cine ha trasmitido sobre la vejez a lo largo de su historia o el papel que tienen las personas de más edad en las diferentes películas. En este artículo simplemente hemos querido hacer una selección intencionada de algunas de ellas. El procedimiento para realizar la búsqueda de las películas a analizar fue el siguiente. En primer lugar, se realizó una búsqueda a partir de las bases de datos más conocidas en materia de cine (www.imdb.com,www. imbd.es, www.filmotech.com, por ejemplo) y en páginas web dedicadas a la crítica de cine www.filmin.es, www.blogdecine.com, www.filmaffinity.com, www.labutaca.net, www.mundocine.com). A través del Google se encontraron otras películas al introducir algunas palabras clave (entre otras, "vejez y cine", "mayores en el cine", "amor maduro en el cine", "relaciones intergeneracionales y cine", "Alzheimer y cine" o "cine y final de la vida"). Se buscó la filmografía reciente de algunos actores conocidos y que ya superan los 60 años en Wikipedia (www.wikipedia.com) y en algunas páginas dedicadas al cine. Además y finalmente, se realizaron entrevistas a expertos y críticos de cine que aconsejaron incluir algunos films en el análisis, los que ellos denominaron "películas imprescindibles". Tras el visionado y análisis de 225 de películas que tratan el tema de la vejez, el paso del tiempo o sus protagonistas son personas mayores de 55 años, realizamos una selección de 49 películas que hemos clasificado a partir del tema principal que abordan si bien muchas de las películas tratan el tema de la vejez desde diversas perspectivas, cruzándose en ellas diversos aspectos como la jubilación, la perpetuación de un legado, el repaso de lo que ha sido la vida y el sentido de trascendencia, las relaciones afectivo-sexuales o la soledad en la vejez, la noticia del final de la vida y la muerte.

El primer cribado permitió dividir las películas visionadas en aquellas que hablan de envejecimiento activo o muestran una visión positiva de la vejez y el envejecimiento y hablan también de la solidaridad intergeneracional (tabla 1) y aquellas otras que muestran un envejecimiento patológico, una visión negativa de la vejez o hablan de los cuidados en la dependencia (tabla 2).

Tabla 1. Vejez activa. Visión positiva del envejecimiento. Adaptación positiva

\section{Vejez activa.}

Visión positiva de la vejez y el envejecimiento Solidaridad intergeneracional

Adios Mr Chips (dir. S Wood, 1939)

Ahora o Nunca (dir.R Reiner, 2007)

Al otro lado (dir.FAkin, 2007)

Anotheryear (dir. M Leigh, 2010)

Antonia (dir.M Gorris, 1995)

BurtMunro. Un sueño, una leyenda (dir. R Donaldson, 2005)

Carmina o revienta (dir.P.León, 2012)

Cerezos en flor (dir. D Dorrie, 2008)

Charlie y la fábrica de chocolate (dir. T Burton, 2005)

Cinema Paradiso (dir. G Tornatore, 1988)

Cleopatra (dir. E Mignona, 2003)

Cocoon (dir. R Howard, 1985)

Cocoon. El retorno (dir. D. Petrie, 1988)

Como un Rolling (dir. P. Castilla, 2012). Corto

Conoces a Joe Black? (dir. M Brest, 1998)

Conversaciones con mamá (dir. C. Oves, 2004)

Corazones rebeldes (dir.S Walker, 2007)

Cosas de familia (dir. F. Schepisi, 2008)

Cuando el viento sopla (dir. J. T Murakami, 1986)

Cuando Harry conoció a Sally (dir. R. Reiner, 1989)

Cuando menos te lo esperas (dir. N Meyers, 2003)

Cuéntame una historia (dir. R Donna, 2004)

Cuentos de Tokyoldir. Y Ozu, 1953)

Dame 10 razones (dir. B. Silberling, 2006) 
Dejad paso al mañana (dir. L McCarey, 1937)

Del rosa al amarillo (dir. M Summers, 2003)

Dersu Uzala (dir. A. Kurosawa, 1975)

Descubriendo a Forrester (dir. G Vas Sant, 2000)

Despedidas (dir. Y. Takita,2008)

Donde reside el amor (dir. J Moorhouse, 1995)

Ed Wood (dir. T. Burton, 1994)

El abuelo (dir. JL. Garci, 1998)

El artista y la modelo (dir. F. Trueba, 2012)

El bosque del luto (dir. N Kawase, 2007)

El color del paraíso (dir.M.Majidi, 1999)

El coronel no tiene quien le escriba (dir. A Ripstein, 1999)

El estanque dorado (dir. M. Rydell, 1981)

El estudiante (dir. R Girault, 2009)

El exótico hotel Marigolddir. J Maden, 2011)

El fin es mi principio (dir. J Baier, 2010)

El nido vacío (dir. D Burman, 2008)

El oro de Ulises (dir.V Nuñez, 1997)

El prado de las estrellas (dir. M Camus, 2007)

El regalo (dir. C. Galaz, 2008)

El reto (dir. A Aguilar, 2009)

El rey de las máscaras (dir. W Tianming, 1997)

El rey Lear (dir. M Radford, 2012)

El rio de la vida (dir. R Redford, 1992)

El secreto de los McCann (dir.T McCanlies, 2005)

El señor Ibrahim y las Flores del Corán (dir. F Dupeyron, 2003)

El último (dir. FW Murnau, 1924)

El último pistolero (dir. D. Siegel, 1976)

El verano de Kikujiro (dir. T. Kitano, 1999)

El viaje de nuestra vida (dir. C.N. Rowley, 2006)

El viejo y el mar (dir. J Sturges, 1958)

El viento (dir. E Mignona, 2005)

El violín (dir. F Vargas, 2005)

Elsa y Fred (dir. M Carnevale, 2005)

En 80 días (dir. JM. Goenaga, 2010)

En el estanque dorado (dir. M Rydell, 1998)

En el séptimo cielo (dir. A Drese, 2008)

Esencia de mujer (dir. M Brest, 1992)

Espera un poco (dir.A Kurosawa, 1993)

Frankie y Johnydir. G Marshall, 2001)

Geri's game (dir. J. Pinkava, 1997) Corto.

Gianni y sus mujeres (dir. G di Gregorio, 2011)

Gran Torino (dir. C Eastwood, 2008)
Haciendo tiempo para volver a casa (dir. G. Jordan, 1997)

Harold and Maud (dir. H. Ashby, 1971)

Harry and Tonto (dir, P.A Mzursky, 1971)

Innocence (dir. P Cox, 2000)

Intimo y personal (dir. J Avnet, 1996)

Ironias de la vida (dir. A Aguilar, 2006)

Kolyaldir. J Severak, 1997)

Kotch (dir. J. Lemmon, 1971)

La casa de mi abuela (dir. A Aliaga, 2006)

La dama y la muerte (dir. J. Recio, 2009) Corto.

La escalera (dir. S Donen, 1969)

La muñeca del espacio (dir. D Moncasi, 2006).

La pequeña habitación (dir. M Bouquet, 2012)

La soledad (dir. J Rosales, 2007)

La última estación (dir. M Hoffman, 2009)

La última primavera (dir. C Dance, 2004)

La ventana (dir. C Sorin, 2008)

La vida empieza hoy (dir. L Maña, 2009)

Las chicas de la lencería (dir. B Oberli, 2006)

Las chicas del calendario (dir. N Cole, 2003)

Las horas del verano (dir. 0 Assayas, 2008)

Lola (dir. B Mendoza, 2009)

Lugares comunes (dir. A Aristaráin, 2002)

Mamá cumple 100 años (dir.C Saura, 1979)

Mi padre (dir. G.D. Goldberg, 1989)

Mia Sarah (dir. G Ron, 2006)

Mil años de oración (dir. W Wang, 2007)

Million Dollar Baby (dir. C Eastwood, 2004)

Mis tardes con Margueritte(dir. J Becker, 2009)

Mr. Henderson presenta (dir. S Frears, 2005)

Nietos. Identidad y memoria (dir. B. Avila, 2003)

Nunca es tarde para enamorarse (dir. J Hopkins, 2009)

Otoño en Nueva York (dir. J Chen, 2000)

Para que no me olvides (dir. P Ferreira, 2005)

Paseando a Miss Daisy (dir. B Beresford, 1989)

Pequeño Cherokee (dir. R. Friedenberg, 1997)

Poesía (dir. L Chang-Dong, 2010)

Por si no te vuelvo a ver (dir. JP Villaseñor, 2000)

Primavera en otoño (dir.C Eastwood, 1973)

Primavera, verano, otoño, invierno... y primavera (dir. K.

Ki-Duk, 2003)

Profesor Holland (dir. S.Herek, 1995)

Regreso a Bontiful (dir. P Masterson, 1985)

Relámpago sobre el agua (dir. W Wenders, 1980)

Robin y Marian (dir. R Lester, 1976) 
Romance otoñal (dir. B Kidron, 1992)

Sang Woo y su abuela (dir. L. Jeong-Hyang, 2002)

Saraband (dir. I. Bergman, 2003)

Si de verdad me quieres (dir. D. Frankel, 2012)

Siempre hay tiempo (Héctor y Bruno)(dir. A Diego, 2009)

Sin perdón (dir. C Eastwood, 1992)

Sol de otoño (dir. E Mignona, 1996)

Solas (dir. B Zambrano, 1999)

Solo el cielo lo sabe (dir. D Sirk, 1955)

Son of Babylon (dir. M. Al Daradji, 2009)

Sonata de otoño (dir. I Bergman, 1978)

Sostiene Pereira (dir. R Faenza, 1996)

Space cowboys (dir. C Eastwood, 2000)

Tess y su guardaespaldas (dir.H Wilson, 1994)

The mother (dir. R Michell, 2000)

The visitor (dir. T. McCarthy, 2007)

Tierras de penumbra (dir. R Attemborough, 1993)

Todos están bien (dir. K Jones, 2009)

Tomates verdes fritos (dir. J. Avnet, 1991)

Tres veces 20 años (dir. J Gravas, 2011)

Umberto $D$ (dir. V. de Sica, 1952)

Un año más (dir. M Leigh, 2011)

Un buen día lo tiene cualquiera (dir. S Lorenzo, 2007)

Un poco de chocolate (dir. A. Aramaio, 2008)

Un toque de canela (dir.T. Boulmetis, 2003)

Una dama digna (dir. P.T. Anderson, 1999)

Una historia verdadera (dir. D. Lynch, 1999)

Una vida por delante (dir. L. Hallström, 2005)

Up (dir. B. Peterson, 2009)

Venus (dir. R Mitchell, 2006)

Vivir(dir. A. Kurosawa, 1952)

Volver a empezar (dir. J.L. Garci, 1982)

Vuelvo a casa (dir. M Olivera, 2001)

Zapatos italianos (dir. K Branagh, 2014)

Tabla 2. Vejez patológica. Visión negativa del envejecimiento. Cuidados en la dependencia

\section{Vejez patológica.}

Visión negativa de la vejez y el envejecimiento.

Cuidados den la dependencia.

A propósito de Schmidt (dir. A. Payne, 1993)

Acordarse de cosas bellas (dir. Z Breitman, 2001)

Age old friends (dir A. Kroeker, 1989)
Algo queda (dir. A. Lorenz, 2010).Corto

Amanecer de un sueño (dir. F. Mas Franqueza, 2008)

Amour (Love) (dir. M. Haneke, 2012)

Arrugas (dir. I Ferreras, 2011). Corto animación

Aurora borealis (dir. CE Burke, 2006)

Bicicleta, cuchara, manzana (dir. C. Bosch, 2010).

Bucarest, la memoria perdida (dir. A. Sole, 2009).

Club eutanasia (dir. A Tapia, 2005)

Colgadas (dir. D. Keaton, 2000)

Complaints of a dutiful daughter (dir. D Hoffmann, 1994)

La comunidad (dir. A. de la Iglesia, 2000)

La escapada (dir. D. Risi, 1962)

La extraña pareja (dir. G. Saks, 1968)

La extraña pareja otra vez (dir. H. Deutch, 1998)

La familia, bien, gracias (dir. Masó, 1979)

La familia, 30 años después (dir. P Masó, 1999)

La familia Savages(dir. T. Jenkins, 2007)

La grandeza de vivir(dir. A Byrne, 2007)

La hora del adiós (dir. D. Jones, 1997)

La luna en el espejo (dir. S Caiozzi, 1990)

Las manos de la madre (dir. M. Gabilondo, 2013)

La memoria del asesino (dir. E Van Looy, 2003)

La pareja chiflada (dir. H Ross, 1975)

La primavera romana de la Sra. Stone (dir. J. Quintero, 1961)

La ventana abierta (dir. D. Cañizal, 2010)

La vieja de atrás (dir. P.J. Meza, 2010)

Las ballenas de agosto (dir. L Anderson, 1987)

Las invasiones bárbaras (dir. D. Arcand, 2003)

Lejos de ella (dir. S Polley, 2006)

Las voces de la memoria (dir. V Peris, DFabra, A Badia, 2011)

Los montes (dir. J.J. Martin, 1982)

Magnolia (dir. P.T. Anderson, 1999)

Nedar (dir. C Subirana, 2008)

Poesía (dir. L Chang-Dong, 2010)

Primavera tardía (dir. Y Ozu, 1949)

¿Qué fue de Baby Jane? (dir. R Aldrich, 1962)

¿Qué hacemos con la abuela? (dir. E Chatilliez, 1990)

Sacrificios del corazón (dir. DS. Cass, 2007)

¿Te acuerdas del amor? (dir. J Bleckner, 1985)

Un buen día lo tiene cualquiera (dir. S Lorenzo, 2007)

Un camino hacia la luz (dir. R.A. Acckerman, 1999)

Una canción para Marthy (dir. B August, 2001) 
Volver a Villarro (dir. J.J. Perez, 2005)

¿Y tú quién eres? (dir. A Mercero, 2007)

Zombie (dir. D Moreno, 2012)

Esto no es un cuento (dir. L Aubia, 2012)

Eternamente joven (dir. S. Miner, 1992)

Fedora (dir. B Wilder, 1978)

Fresas salvajes (dir. I Bergman, 1957)

Firefly dreams (dir. J Williams, 2001)

Ginger y Fred (dir. F Fellini, 1985)

Hasta mañana (dir. L Pedraz, 2010)

Historias mínimas (dir. C Sorin, 2002)

Iris (dir. R Eyre, 2001)

Juguetes rotos (dir. M Summers, 1966)

Justino, un asesino de la tercera edad (dir.S Aguilar, 1994)

La balada de Narayama (dir. S. Imamura, 1983)

La caja de Pandora (dir. Y. Ustaoglu, 2008)

Cortex (dir. N Boukhrief, 2008)

Cosas que importan (dir. C. Franklin, 1998)

Cuidadores (dir. 0 Tejedor, 2010)

Discordias a la carta (dir. H. Deutch, 1995)

Dos viejos gruñones (dir. D Petrie, 1993)

Dos viejos más gruñones (dir. H Deutch, 1995)

El cochecito (dir. M Ferreri, 1960)

El crepúsculo de los dioses (dir. B Wilder, 1950)

El diario de Noah (dir. N Cassavetes, 2004)

El hijo de la novia (dir.J.J.Campanella, 2001)

El mundo de Marti (dir. D Bardiau, 2000)

El sabor del sake (dir. Y Ozu, 1962)

El viejecito (dir. M Summers, 1959)

En la ciudad sin límites (dir. A Hernandez, 2001)

Memories of tomorrow (dir. Y Tsutsumi, 2006)

Mitio Jacinto (dir. L Vajda, 1956)

Muerte en Venecia (dir. L Visconti, 1971)

Nader y Simin, una separación (dir. A. Farhadi, 2011

En el presente artículo nos centramos sólo en aquellas que hablan del envejecimiento activo y la solidaridad intergeneracional, la vejez como momento de cambio y crecimiento, el envejecimiento en positivo, el afrontamiento y superación de los problemas que la vida plantea, dejando para un artículo posterior el análisis de otras temáticas también interesantes para los psicólogos o educadores en general y para los gerontólogos en particular, como el cuidado, la dependencia, el impacto del cuidado en la familia o el afrontamiento del final de la vida independiente. En otro artículo nos ocuparemos de cómo proceder para un uso didáctico de estas películas, sugiriendo cómo poner en marcha un programa de cine centrado en el diálogo intergeneracional que ayude a cambiar los estereotipos negativos asociados a la vejez y promueva un acercamiento entre generaciones.

Un equipo compuesto por cinco miembros (tres expertos en gerontología y asesorados por especialistas en cine) visionó las diferentes películas y les fue asignando de modo independiente unas etiquetas que pudiesen después dar lugar a una primera clasificación temática. Las películas fueron etiquetadas como: envejecimiento activoenvejecer en positivo, la vida después de la jubilación, la transmisión de un legado/ la mentorización, las relaciones intergeneracionales (entre abuelos/nietos y entre personas de diferentes edades), la superación del duelo y el afrontamiento positivo, incluida la aceptación de la proximidad de la muerte.

Tras una puesta en común de las etiquetas y por un procedimiento de consenso máximo, se llegó a la siguiente clasificación de temas y subtemas.

a. Cambios, acontecimientos vitales, cambios sociales, pero también el modo de responder a dichos cambios (afrontamiento positivo, adaptación, resiliencia), integridad: jubilación, nido vacío, proximidad a la muerte propia o ajena, viudedad. 
b. Relaciones sociales, salud psicológicaemocional, relaciones afectivo-sexuales

c. Relaciones familiares, relaciones padres que envejecen e hijos adultos

d. Relaciones intergeneracionales y generatividad. Mentorización. Transmisión de un legado. Personas mayores como educadores. Relación abuelos-nietos

e. Participación activa y generatividad. Participación social, cultural, educativa, aprendizaje a lo largo de la vida.

\section{Una visión psicosocial incompleta...}

Cada película se ha incluido en una categoría en la que mejor representada está, pero lo cierto es que la mayor parte de ellas hablan de diversos temas o diferentes personajes, podrían enmarcarse en distintos modos de envejecer, mostrándose en muchas de ellas el contraste entre dos o más personajes o el cambio de un personaje (o varios) del comienzo al final del film.

Tabla 3. Películas seleccionadas y principales temas y subtemas tratados en ellas

\begin{tabular}{|c|c|c|c|}
\hline \multirow{6}{*}{  } & \multirow{6}{*}{$\begin{array}{l}\text { 1.CAMBIOS-ACONTECIMIENTOS VITALES. } \\
\text { CAMBIOS SOCIALES } \\
\text { pero también el modo de responder a } \\
\text { dichos cambios: } \\
\text { SALUD PSICOLÓGICA-EMOCIONAL. } \\
\text { AFRONTAMIENTO POSITIVO/INTEGRIDAD } \\
\text { MODELO OPTIMIZACIÓN SELECTIVA POR } \\
\text { COMPENSACIÓN } \\
\text { La vida empieza hoy } \\
\text { INTEGRIDAD } \\
\text { Una historia verdadera } \\
\\
\text { 2. RELACIONES SOCIALES. RELACIONES } \\
\text { FAMILIARES. SALUD PSICOLÓGICA- } \\
\text { EMOCIONAL. }\end{array}$} & 1.1. JUBILACIÓN & $\begin{array}{l}\text { A propósito de Schmidt } \\
\text { Vivir }\end{array}$ \\
\hline & & 1.2 VIUDEDAD & $\begin{array}{l}\text { Cerezos en flor } \\
\text { El bosque del luto }\end{array}$ \\
\hline & & 1.3. NIDO VACÍO & El nido vacío \\
\hline & & $\begin{array}{l}\text { 1.4. PROXIMIDAD DE LA PRO- } \\
\text { PIA MUERTE. LA MUERTE }\end{array}$ & $\begin{array}{l}\text { Volver a empezar } \\
\text { Ahora o nunca } \\
\text { Fresas salvajes } \\
\text { Los montes } \\
\text { Despedidas }\end{array}$ \\
\hline & & $\begin{array}{l}\text { 2.1.RELACIONES AFECTIVO- } \\
\text { SEXUALES }\end{array}$ & $\begin{array}{l}\text { Amour (Love) } \\
\text { Elsa y Fred } \\
\text { En el séptimo cielo } \\
\text { En } 80 \text { días } \\
\text { La vida empieza hoy } \\
\text { Zapatos italianos } \\
\text { The mother } \\
\text { Tres veces } 20 \text { años }\end{array}$ \\
\hline & & $\begin{array}{l}\text { 2.2.RELACIONES PADRES QUE } \\
\text { ENVEJECEN- HIJOS ADULTOS }\end{array}$ & $\begin{array}{l}\text { Cerezos en flor } \\
\text { Conversaciones con mamá } \\
\text { Están todos bien } \\
\text { Cuentos de Tokyo } \\
\text { Dejad paso al mañana } \\
\text { Mama cumple cien años }\end{array}$ \\
\hline
\end{tabular}




\begin{tabular}{|c|c|c|c|}
\hline \multirow{4}{*}{ 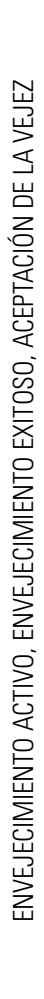 } & \multirow[b]{2}{*}{$\begin{array}{l}\text { 3.RELACIONES INTERGENERACIONALES } \\
\text { y también } \\
\text { GENERATIVIDAD } \\
\text { El señor Ibrahim y las Flores del Corán } \\
\text { Million Dollar Baby } \\
\text { SABIDURÍA } \\
\text { Tomates verdes fritos }\end{array}$} & 3.1.ABUELOS-NIETOS & $\begin{array}{l}\text { Charlie y la fábrica de chocolate } \\
\text { Sang Woo y su abuela } \\
\text { Kotch } \\
\text { Vuelvo a casa }\end{array}$ \\
\hline & & $\begin{array}{l}\text { 3.2.MENTORIZACIÓN. } \\
\text { TRANSMISION DE ENSE- } \\
\text { ÑANZAS y/o AYUDA de una } \\
\text { generación a otra } \\
\text { 3.3.SOLIDARIDAD INTERGE- } \\
\text { NERACIONAL }\end{array}$ & $\begin{array}{l}\text { Descubriendo a Forrester } \\
\text { El señor lbrahim y las Flores del Corán } \\
\text { Million Dollar Baby } \\
\text { Cinema Paradiso } \\
\text { Tomates verdes fritos } \\
\text { Pequeño cherokee } \\
\text { Esencia de mujer } \\
\text { El bosque del luto } \\
\text { Antonia } \\
\text { Mis tardes con Margueritte } \\
\text { Un poco de chocolate } \\
\text { Up } \\
\text { Una dama digna } \\
\text { El fin es mi principio }\end{array}$ \\
\hline & \multirow[b]{2}{*}{ 4.PARTICIPACIÓN ACTIVA } & 4.1.SOCIAL, CÍVICO-POLÍTICA & $\begin{array}{l}\text { Conversaciones con mamá } \\
\text { Por si no te vuelvo a ver }\end{array}$ \\
\hline & & $\begin{array}{l}\text { 4.2.CULTURAL-EDUCATIVA- } \\
\text { FORMATIVA }\end{array}$ & $\begin{array}{l}\text { El estudiante } \\
\text { Poesía } \\
\text { Como un Rolling } \\
\text { Corazones rebeldes }\end{array}$ \\
\hline
\end{tabular}

Además del criterio primero (películas o cortos que muestren un afrontamiento positivo al envejecimiento o hablen del envejecimiento activo), hemos querido seleccionar películas de diferentes géneros (drama, comedia, animación, documental, biográfica, romántica), de diferentes épocas (desde 1924 a 2013), de distintas nacionalidades (cine japonés, cine americano, cine argentino, cine español) y no olvidar a grandes directores (Eastwood, Lynch, Kurosawa, Oliveira, Ozu, Bergman) y directoras (Dorrie, Kawase) o directores españoles y latinoamericanos (Garci, Mañá, Mignona) ni tampoco a algunos de los grandes actores que se han hecho mayores en el cine. Por eso hemos tratado también de tener este criterio en mente al incluir películas cuyos protagonistas fuesen "actores de largo recorrido" que superan los 60, 70 u 80 años (Manuel Aleixandre -1917-2010-, China Zorrilla -n. 1922-, Hector Alterio -n. 1929, Sean Connery -n.1930-, Clint Eastwood -n.1930-, Omar Sharif -n.1932-, Robert Redford -n.1936-, Jack Nicholson -n.1937-, Anthony Hopkins -n.1937-, Morgan Freeman -n.1937-, Helen Mirren -n.1945-, Merryl Streep -n.1949-, Isabella Rossellini -n.1952-). Ya sabemos que nos dejamos a muchos otros en esta selección, ejemplos de buen envejecer: Michael Caine, Olympia Dukakis, Lauren Bacall, Vanessa Redgrave, Christopher Lee, Peter O'Toole, Eli Wallach, Richard Harris, Judi Dench, Diane 
Keaton, o Glen Close. Somos conscientes de que hay muchas más, incluso algunas que el/ la lector/a podría valorar como mejores películas de las aquí presentadas y por eso añadimos el listado de las películas visionadas y revisadas y declaramos este artículo como inacabado pero también abierto.

\section{Referencias}

Baltes, P. y Baltes, M.M. (1990). Psychological perspectives on success fulaging: The model of selective optimization with compensation. En P.B. Baltes y M.M. Baltes (Eds.), Successful aging: Perspectives from the behavioural sciences (pp. 1-34). New York: Cambridge University Press.

Baltes, P.B. y Smith J. (2003). New frontiers in the future of aging: From successful aging of the young old to the dilemmas of the fourth age. Gerontology, 49, 123-135.

Baltes, P.B. y Staudinger, U.M. (2000). Wisdom. A metaheuristic (pragmatic) to orchestrate mind and virtue toward excellence. American Psychologist, 55, 122-136.

Baltes, P.B., Smith, J., Staudinger, U.M., y Sowarka, D. (1990). Wisdom: One facet of successful aging? En M. Perlmutter (Ed.), Late-lifepotential (pp. 63-81). Washington, DC: GerontologicalSociety of America.

Butler, R. (1969). Ageism: Another form of bigotry. The Gerontologist, 9: 243-6.

Castells,M.(2000): The information age: economy, society and culture. Vol 1: The rise of the network society, 2nd. edition. New York: Blackwell.

Diputación Foral de Vizcaya (2011), Las personas mayores y los Medios de Comunicación. Bilbao: Diputación Foral de Vizcaya, Departamento de Acción Social.

Erikson, E. (1971). Identidad, juventud y crisis. Buenos Aires: Paidós

Erikson, E. y Erikson, J.M. (1987/2000). El ciclo vital completado. Barcelona: Paidós.

Estrategia de Lisboa -Plan Bolonia- (1999). Recuperado en: http://www.mecd.gob.es/boloniaeees/inicio.html

Genovard, C. y Casulleras, D. (2005). La imagen de la vejez en el cine. Iconografía virtual e interpretación psicológica. Boletín de Psicología, 83, 7-20.

Gómez, P. y Díaz-Veiga, P. (2011) (coor.), Guía Práctica del Buen Trato a las personas mayores. Madrid: Sociedad Española de Geriatría y Gerontología.

Hannah, M.T., Domino, G., Figueredo A.J., y Hendrickson, R. (1996). The prediction of ego integrity in older persons. Educational and Psychological Measurement, 56:930-950.

http://ecodiario.eleconomista.es/musica/noticias/2521914/10/10/Ronnie-Wood-Los-Rolling-Stonesno-se-separaran-hasta-que-todos-los-miembros-hayanfallecido.html

INE (2012). Recuperado en: www.ine.es

Informe al Parlamento Europeo sobre el futuro demográfico de Europa 2007/2156. Recuperado en:

http://www.europarl.europa.eu/oeil/popups/ficheprocedure.do?lang=fr\&reference $=2007 / 2156$ (INI)

Ley del Cine (Ley 55/2007, de 28 de diciembre). Recuperado en: http://www.boe.es/boe/dias/2007/12/29/pdfs/ A53686-53701.pdf

Ley Orgánica de Educación (Ley Orgánica 2/2006, de 3 de mayo). Recuperado en: http://www.boe.es/boe/ dias/2006/05/04/pdfs/A17158-17207.pdf

OMS (2002). Active aging. A policy framework. Recuperado en: http://whqlibdoc.who.int/hq/2002/who_nmh_ nph_02.8.pdf

Ortiz, A. (2006). El cine y la edad. En S. Pinazo (dir), El viatge de la Nau Gran. Valencia: Publicacions de la Universitat de Valencia.

Ramos, I. y Carretón-Ballester, MC (2012), Presencia y representación de las personas mayores en la publicidad televisiva: el caso español. Revista Española de Geriatría y Gerontología, 47, 2, 55-61.

Triadó, C. y Villar, F. (2008). Envejecer en positivo. Gerona: Aresta

UNESCO (1960). Segunda Conferencia Mundial de Educación de Adultos. Recuperado en: http://unesdoc. unesco.org/images/0013/001338/133863so.pdf

Fecha de recepción: 24/01/2013

Fecha de aceptación: 20/02/2013

Nota de la autora:

Hablando de personas mayores y cine...

...quiero expresar mi agradecimiento más sincero a un grande del cine, Adolfo Bellido, por sus muy buenos consejos, por los ratos que hemos pasado hablando de cine y por lo mucho que he aprendido a su lado, de cine y de la vida.

...también a Ramón Bayés, que es un Maestro, en esto de utilizar el cine para hablar de Psicología y en muchas más cosas. 